\title{
Web-based Platform for Psychology Research
}

\author{
Ilya Zakharov ${ }^{1}$, Evgeny Nikulchev ${ }^{2}$, Dmitry Ilin $^{2,}{ }^{3}$, Victoria Ismatullina ${ }^{1}$, and Alexander Fenin ${ }^{1}$ \\ ${ }^{1}$ Psychological Institute of Russian Academy of Education, 125009 Moscow, Russia \\ ${ }^{2}$ Moscow Technological Institute, 199334, Moscow, Russia \\ ${ }^{3}$ Moscow Technological University MIREA, 119454 Moscow, Russia
}

\begin{abstract}
This article is devoted to contemporary methods of risk management under the conditions of crisis on financial markets. The article deals with developing the new approaches to studying finance and financial markets. It outlays new methods to compare fundamental events and their technical interpretation shown on quotation charts. It also introduces recommendations as to improve the risk management's efficiency of financial markets' participants.
\end{abstract}

\section{Introduction}

During the last few decades psychological research is consistently moving from laboratories to internet and web-based studies. Web-technologies offer numerous advantages for psychologists. For example, according to Andrew Gelman and colleagues [1], psychological studies tend to be systematically underpowered, which is stated as one of the reasons for the replication crisis in psychology. An easy access to bigger samples through internet and social networks provides an opportunity to radically enlarge the study samples thus leading to more reliable results. However, web-technologies offer more than just an increase in the numbers of people involved. Another advantage is the possibility to individualize the outcome and feedback for participants. The amount of data is getting big enough to start using machine learning and other artificial intelligence approaches. In the present article we will review existing web-based technologies for psychological research around the world with an emphasis on the situation in Russia.

\section{Analysis of platforms for psychological research}

The first widely used web-based psychological tool was the "Amazon Mechanical Turk" (MTurk, [2]). It was a crowdsourcing internet marketplace where researchers were able to recruit subjects for taking part in online studies. Subjects got a small amount of money for participating. MTurk was used mostly by researchers from the US. It was shown that MTurk samples did not perfectly match the US population characteristics. For example, MTurk population was mostly white and female, and more educated and young than overall US population. However, the quality of the obtained data remained good enough [3]. MTurk was successfully used to study attention [4], creativity and dishonest behavior
[5] or sexual attitudes [6]. It has been shown that MTurk can be a valuable tool even for working with clinical population [7] (Shapiro et al., 2013).

After the MTurk success new technologies started to appear. However, the framework has changed. While MTurk was a marketplace that accumulated its own audience, new tools only provided instruments to develop studies that could then be distributed through the web, but it was the responsibility of the researcher to find the sample. One of the most famous tool of that type became Google Forms (Google Inc.). This free software was intended to create web-surveys. Every researcher was able to create his questionnaire for which he was given a unique link that he was able to spread elsewhere in the internet. Google Forms also provided some simple analytics for the researchers. Its openness and Google brand made it ubiquitous for online surveys, especially among students. However, Google Forms lack some of the features that play an important role in psychological research. For example, the analysis of the amount of time spent for each part of the survey is hardly feasible with the Google Forms. There are also some concerns regarding the way the data is stored. To face the needs of the psychological society, some new products started to appear. Out of the most recognized, we can mention Survey Monkey [8], Qualtrics [9], LimeSurvey [10] or EnKlikAnketa [11]. Some of these products are proprietary or at least shareware (SurveyMonkey, Qualtrics), some are free (EnKlikAnketa).

These services tend to provide many new functions for researchers. The Slovenian EnKlikAnketa shows one of the non-mainstream way to improve online services. For example, it offers to fix the methodological flaws in the design of the surveys. Apart from being completely free, it has plenty of other useful advantages that are not implemented in other systems. First of all, it collects a lot of metadata, such as which site the respondent came from, how much time he or she spent in the survey and 
how many pages he actually visited, what browser and system he or she had and many other indicators. Secondly, it allows a much broader variety of item types, advanced preferences for every item (such as choosing the numeric/ordinal scale, showing statistics for this item to the respondent, returning the feedback after in-system computing etc.) and scenarios based upon the if-then scripts for several choices. Moreover, $1 \mathrm{ka}$ allows its users to save, copy and share questions through its library system, thus saving a lot of time that would be otherwise spent on rewriting the questions and categories. Finally, it has a wide range of built-in analytic instruments, computing frequencies, sums, contingency tables and much more, as well as a graphic constructor, that enables researchers to either generate fulldata descriptive statistics in several clicks or to flexibly adjust the diagrams for specific data reporting. Finally, $1 \mathrm{ka}$ allows the data export in many formats, including .sps for SPSS, that is widely used by psychologists around the world.

Today, these services have almost replaced the traditional pen-and-paper studies. However, they can be used only for the questionnaires or knowledge-type tests (q-type of data according to Cattell [12] ) thus ignoring some areas of currently challenging psychological topics, such as, for example, individual differences in cognitive or executive functions. In these areas the modern technologies have already replaced the classical pen-and-paper approach. Here, the computerized automated tools are being developed [13, 14]. Computerized adminsitration of tests is convenient due to several factors. Firstly, it helps to monitor the administration process automatically thus reducing the errors related to human factor. Secondly, computerized tools of this type can record the aspects of performance with the precision that is difficult to achieve otherwise, and "these may reflect activity in developing neural networks with more sensitivity than can be achieved with traditional tests" [15].

Different cognitive tests are organized in batteries that are designed to assess the cognitive sphere integrally. The widely used cognitive characteristics include general cognitive abilities, working memory [16], spatial or mathematical abilities [17], recognition processes [18], and many others. Most of these batteries have been developed for the clinical use (NAB, CNTB, $[19,20])$. However, they have also been successfully used to study the normative sample $[15,21,22]$. We have also showed its advantages in the behavioral genetics studies [23].

Cognitive tests are becoming more and more available to researchers. First, there are free software solutions, such as PsychoPy [24], that allows psychologists to develop their own tests without the need for advanced programming skills. Some of the applications of that type even include their own batteries of test. For example, though the Psychology Experiment Building Language (PEBL, [25]) requires more skills to create the test, it already has a large preprogrammed set of executables that allow to measure a wide range of characteristics.
Currently, the main limitation of the automated batteries is that they can only be administered offline with downloaded and pre-installed software. The psychological society tends not to trust the precision of tests administered online because of the potential confounding factors such as the technical properties of the personal computers or monitors of the respondents. However, the quantitative estimation of the noise added due to the differences in setup shows that at least for some type of tasks web-based experiments can be an acceptable source of data [26]. Thus, the next logical step for the computerized cognitive tests is to spread across the internet the same way it has happened to the questionnaires. According to it, the PsychoPy team, for example has recently presented the option to run experiments in a web browser.

The situation with web-technologies in psychological research can differ among the countries. In Russia one of the questions that arise is the localization of the services. The need for localization may concern Russian adaptation of questionnaires as well as translation of the test instructions. The other important factor is related to the data storage, which should be organized according to the local laws. Thus, it is important to develop Russian services that will be able to provide tools to conduct web-based studies. There are already several solutions that can be used (e.g., [27] or [28]) but they are limited to gathering q-type of data.

Web-technologies in psychology are important because they are related not only to the scientific community. According to the Russian federal law №273 and the requirements of the federal education standards, students must be provided with programs adapted to their psychophysiological development and health condition. Thus, educational policy should concern individual, age-related, psychological and physiological characteristics of the students. It is impossible to meet the requirements of the federal law without any continuous psychological assessment. Currently, the ongoing activity has only local nature and the need for the nation-wide projects is on the rise. Bigger scale projects are important because they give an opportunity to build national standards for the diagnostic tools. Webtechnologies may play a key role for addressing these issues.

\section{Architecture of the web-service}

We have already started the project aiming to develop a web-based diagnostic tool for psychological, psychophysiological and cognitive assessment. This tool will combine the power of the online survey systems as well as the automated computerized batteries. It will include a wide range of generally acknowledged psychological methods. Given the rapid development of new methods it will also have the possibility to easily incorporate tools suggested by independent researchers.

From the end-user standpoint, the project will consist of two main components: researcher's private account (fig. 1) and applications for online and offline experiments (fig. 2). 


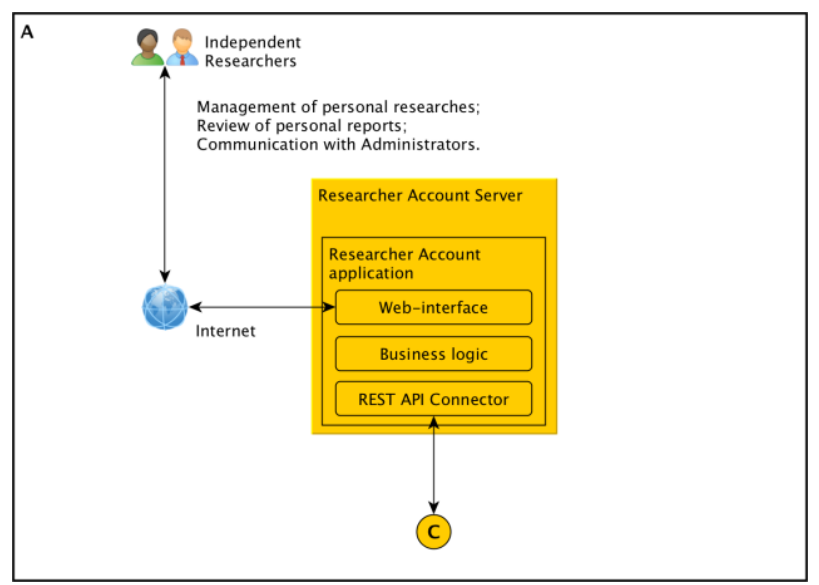

Fig. 1. Independent researcher's tool

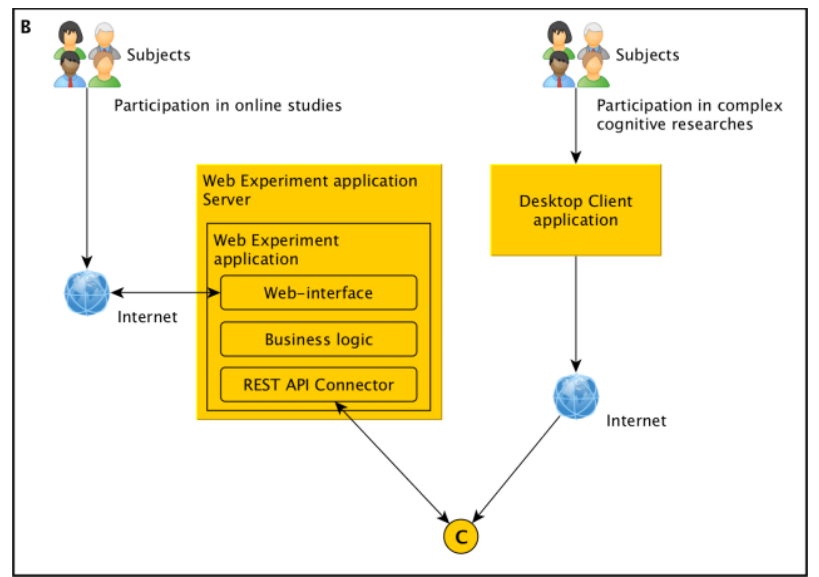

Fig. 2. Online and offline experiment tools

The main priority is the online survey tool as it covers most of possible use cases. Cognitive and psychophysiological assessments apply more requirements to the assessment environment. Thus, it is necessary to develop an offline tool as well.

Considering the fact, that the number of users will grow over time, the service has to be horizontally scalable (fig. 3). It is also essential for surveys with big samples. Each API node should incorporate layered architecture. In combination with the object-document mapping it will provide the service with better flexibility in comparison to monolithic architecture.

Experimental and data intense algorithms for population-base studies should be separated from the main service as well as the administration functionality (fig. 4). In addition, the administration panel can be used as an Intranet service for the security purposes.

In addition to the scaling of algorithmic part, the data storage should be scalable as well. The best fitting approach for the project is combination of sharding and replication (fig. 5). Sharding can provide the system with high I/O performance, while replication can help to ensure the service availability.

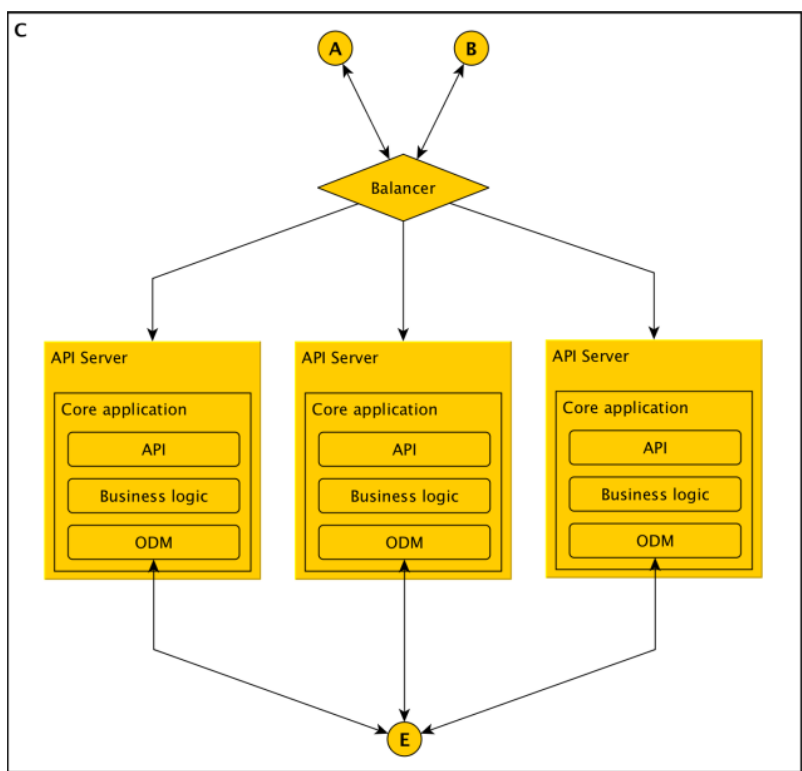

Fig. 3. Scalable API servers for uniform data access and management

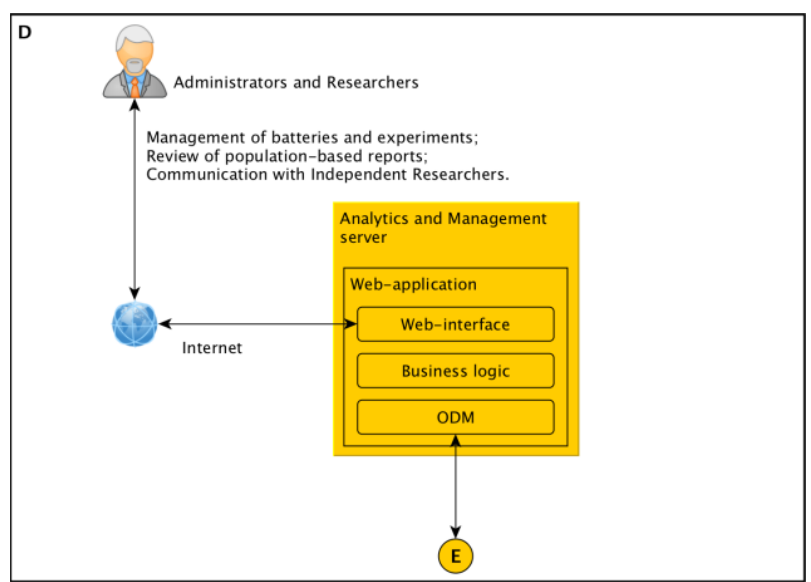

Fig. 4. Isolated analytic tools for population-based researches

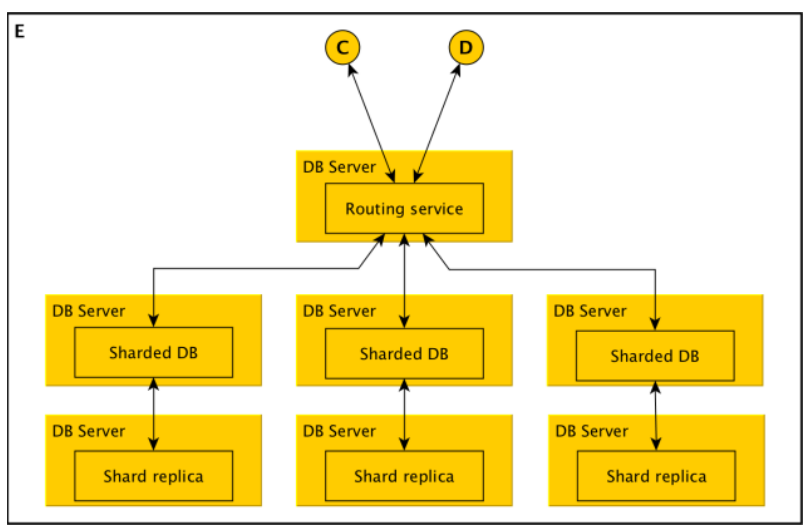

Fig. 5. Horizontal scaling of data storage through sharding

\section{Conclusions}

To conclude, web-based technologies have become much more than just a promising researcher tool. The future development of psychological studies depends on the implementation of new technologies to the same or even greater extent as on the new ideas, and it is 
essential for psychologists in Russia to have their own research tool.

\section{References}

1. http://andrewgelman.com/2016/09/22/why-is-thescientific-replication-crisis-centered-onpsychology/)

2. https://www.mturk.com/mturk/welcome

3. M. Buhrmester, T. Kwang, S.D. Gosling, Perspectives on psychological science, 6, 3 (2011)

4. D.J. Hauser, N. Schwarz, Behavior research methods, 48, 400 (2016)

5. F. Gino, S.S. Wiltermuth, Psychological science, 25, 973 (2014)

6. C. Perilloux, R. Kurzban, Psychological Science, 26, 70 (2015)

7. D.N. Shapiro, J. Chandler, P.A. Mueller, Clinical Psychological Science, 1, 213 (2013)

8. https://www.surveymonkey.com/

9. https://www.qualtrics.com/

10. https://www.limesurvey.org/

11. http://english.1ka.si/

12. R.B. Cattell, Structured personality-learning theory: A wholistic multivariate research approach (Praeger Publishers, 1983)

13. R.M. Bauer, G.L. Iverson, A.N. Cernich et al., Archives of Clinical Neuropsychology, 27, 362 (2012)

14. V. Ismatullina, I. Zakharov, E. Nikulchev, S. Malykh, ITM Web of Conferences, 6, 03005 (2016)
15. M. Luciana, Journal of Child Psychology and Psychiatry, 44, 649 (2003)

16. V. Ismatullina, I. Voronin, A. Shelemetieva, S. Malykh, Procedia-Social and Behavioral Sciences, 146, 353 (2014)

17. I. Zakharov, V. Ismatullina, I. Voronin, S. Malykh, Behavior genetics, 46, 815 (2016)

18. I.M. Zakharov, M. Likhanov, Ju. Marakshina, L. Merzon, K. Poparenko, G.M. Vasin, The proceedings of the International Society for Intelligence Research (ISIR), 98 (2016.)

19. R.A. Stern, T. White, NAB, Neuropsychological Assessment Battery: Administration, Scoring, and Interpretation Manual (Psychological Assessment Resources, 2003)

20. A.E. Veroff, N.R. Cutler, J.J. Sramek et al. Topics in geriatrics, 4, 211 (1991)

21. I.M. Zakharov, I.A. Voronin, V.I. Ismatullina, S.B. Malykh, Procedia-Social and Behavioral Sciences, 233, 313 (2016)

22. I. Voronin, V. Ismatullina, I. Zaharov, S. Malykh SHS Web of Conferences 9, 02043 (2016)

23. I. Voronin, V. Ismatullina, I. Zakharov, G. Vasin, S. Malykh, ITM Web of Conferences, 6, 02010 (2016)

24. https://psychopy.org

25. S.T. Mueller, B.J. Piper, Journal of neuroscience methods, 222, 250 (2014)

26. A. Chetverikov, P. Upravitelev, Behavior research methods, 48, 1086 (2016)

27. http://webanketa.ru/

28. https://virtualexs.ru/ 